\title{
Polyimide-matrix Chemical-selective Membranes
}

\author{
GEUN SIG CHA \\ Department of Chemistry, University of Michigan, Ann Arbor, MI 48109-1055 (U.S.A.) \\ RICHARD B. BROWN \\ Department of Electrical Engineering \& Computer Science, University of Michigan, Ann Arbor, MI 48109-2122 (U.S.A.)
}

\begin{abstract}
Preliminary work using polyimide (PI) as the polymer matrix for permselective membranes is reported in this paper. The excellent adhesion of polyimide may be helpful in extending solid-state chemical sensor lifetimes. Electrochemical performance and adhesion to $\mathrm{Si}_{3} \mathrm{~N}_{4}$ of these membranes is compared to that of conventional PVC-based membranes.
\end{abstract}

\section{Introduction}

Poly(vinylchloride) (PVC) is the most commonly used matrix for permselective polymeric membranes on solid-state ion sensors. One of the primary causes of failure in these microsensors has been poor adhesion of this organic membrane to the chip surface, leading to electrolyte shunts around the membrane which render the sensor inoperative [1]. Improvements by others to membrane adhesion have included modification of PVC for binding to hydroxyl-bearing surfaces $[2,3]$, several methods for mechanically attaching membranes [4-7], and use of other materials [8-10], some of which have improved adhesion but inferior electrochemical performance compared to PVC.

Our research is concerned with both extending sensor lifetime and with achieving mass-fabrication of sensors. These goals have led to development of permselective membranes which use polyimide as a matrix. As demonstrated by Blackburn's suspended-mesh method of attaching membranes of ISFETs [4], polyimide compounds have excellent adhesion to integrated circuit surfaces. Polyimides are also compatible with semiconductor processing, being commonly used as surface protection layers and as dielectrics between metal interconnect layers in integrated circuits. Polyimide films are strong, flexible and insoluble. Conventional polyimide curing (imidization) requires temperatures from 300 to $400^{\circ} \mathrm{C}$, which would evaporate or destroy the ionophore and plasticizer needed to give a membrane the desired electrochemical properties. Fortunately, preimidized polyimide is now commercially available; curing of this material (removal of the solvent) can be accomplished at low temperatures.

Development of polyimide membranes has required particular attention to plasticizers and lipophilic additives. The challenge is to achieve acceptable potentiometric ion response and selectivity while retaining the adhesion properties of polyimide. While the polyimide work is preliminary at this point (membranes have so far been tested electochemically only in ion-selective electrode bodies), based on initial results, we are optimistic about their use in solid-state ion sensors, the target application.

\section{Experimental}

\section{Apparatus}

For all the potentiometric measurements, membranes were mounted in Phillips electrode bodies (IS-561) (Glasblaserei Möller, Zurich). The external reference electrode was an Orion sleeve-type double junction $\mathrm{Ag} / \mathrm{AgCl}$ electrode (Model 9002). Electrodes were connected through a high impedance amplifier to a Zenith Z-100 PC computer equipped with an analog to digital converter (DT 2801, Data Transaction, Inc., Malborough, MA).

\section{Reagents}

Poly(vinyl chloride) high molecular weight (PVC), dibutyl sebacate (DBS), o-nitrophenyl octyl ether (o-NPOE), nonactin, calcium ionophore II (ETH 129), and potassium tetrakis( $p$-chlorophenyl)borate (KTpClPB) were obtained from Fluka (Ronkonkoma, NY). Polyimide (PI; fully imidized and polymerized), and plasticizer sample kit 301 (90 different plasticizers; including dimethyl phthalate (DMP) and diethylene glycol dibenzoate (DGD)) were purchased

(C) Elsevier Sequoia/Printed in The Netherlands 
from Scientific Polymer Products, Inc. (Ontario, NY). Anhydrous tetrahydrofuran (THF; water content $<0.005 \%$ ) and $N, N$-dimethylformamide (DMF; water content $<0.005 \%$ ), were obtained from Aldrich Chemical Co. (Milwaukee, WI); dipentyl phthalate (DPP) from Eastman Kodak (Rochester, NY); and Pyralin LTP (PI-2590-D; preimidized polyimide encapsulant) from DuPont Co. (Wilmington, DE). All other chemicals used were analytical-reagent grade. Standard solutions and buffers were prepared with deionized water.

\section{Preparation of Ion-selective Membranes \\ Polyimide-based ion-selective membranes}

Polyimide (PI) powder was dissolved in DMF $\left(25 \%\right.$, wt./wt.) by heating the solution to $120^{\circ} \mathrm{C}$. Various membranes having different compositions were then prepared by mixing the PI solution with membrane active components (i.e. ionophore, plasticizer and lipophilic additive) and applying the mixture dropwise to a glass plate. The solvent (DMF) was allowed to evaporate for 2 days under vacuum. On the other hand, PI-2590-D polyimide encapsulant was directly mixed with the membrane active components and applied to the glass plate. In this case, longer evaporation periods (4-5 days) were employed due to the high boiling point of the solvent ( $N$-methyl-2-pyrrolidone) used in PI-2590-D.

\section{PVC-based ion-selective membranes}

Ammonium- and calcium-selective membranes were prepared by incorporating nonactin and ETH-129, respectively, into PVC membranes as described elsewhere [11-13].

\section{Evaluating Potentiometric Response}

Small disks were punched from the membranes formed on glass plates, and mounted in Phillips electrode bodies. In the case of the polyimide membranes, a sharp razor was used to detach the membrane from the glass. The inner filling solution varied, depending on which ion-selective membrane was being evaluated: $0.1 \mathrm{M} \mathrm{NH}_{4} \mathrm{Cl}$ for $\mathrm{NH}_{4}{ }^{+}$-selective membranes; and $0.01 \mathrm{M} \mathrm{CaCl}_{2}$ for $\mathrm{Ca}^{2+}$-selective membranes. The calibration plots were obtained from additions of standard solutions to $250 \mathrm{ml}$ of background electrolyte $(0.05 \mathrm{M}$ Tris- $\mathrm{HCl}, \mathrm{pH} 7.2)$ at room temperature. Selectivity coefficients were determined by the separate solution method [14].

\section{Evaluating Adhesion Characteristics}

Adhesion characteristics were evaluated using the method described by Moody et al. [3]. For each membrane type, ten membranes were formed by dispensing drops of membrane mixture onto a silicon wafer coated with $10000 \AA$ of plasma- deposited silicon nitride. In the case of PI membranes, an adhesion promoter (VM-651, DuPont Co., Wilmington, DE) was applied to the wafer before casting the membranes. After the membranes were cured, the wafers were immersed in an ultrasonic bath (Branson Cleaning Equipment, Smithkline Co., Shelton, CN). The time to detachment was recorded for each membrane.

\section{Results and Discussion}

In our initial work with polyimide-matrix membranes, it was found that the plasticizers frequently used for the preparation of PVC-based ion-selective membranes (e.g., DBS, DOA, oNPOE, etc.) were not compatible with polyimide. Among more than 90 plasticizers tested for incorporation in polyimide matrices, only two, dimethyl phthalate (DMP) and diethylene glycol dibenzoate (DGD), resulted in transparent, flexible, yet mechanically strong membranes. Incorporating these plasticizers into the polyimide matrix appeared to have little effect on the excellent adhesion properties of the resulting membranes toward glass surfaces. These membranes cannot be pulled free from the glass, but must be cut from the plate with a razor blade.

In order to examine the feasibility of using polyimide as the matrix of ion-selective membranes, nonactin $(2 \mathrm{mg})$ was incorporated into polyimide matrix membranes ( $75 \mathrm{mg}$ of PI) along with a plasticizer DMP or DGD $(130 \mu l)$. Figure 1 illustrates the potentiometric response of the resulting PI-based membranes toward ammonium ions. As can be seen, the PI membrane prepared with DGD exhibited better response characteristics (i.e. lower detection limit) than that prepared with DMP. However, the response characteristics of the PI/DGD-based ammonium-selective mem-

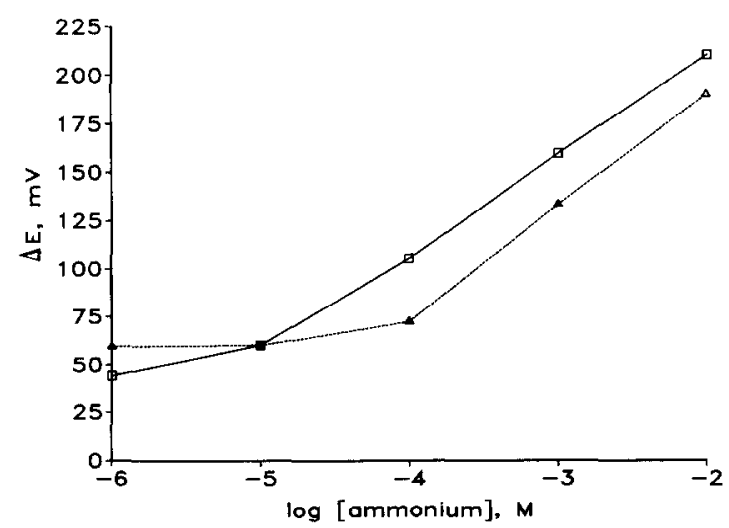

Fig. 1. Potentiometric ammonium response of PI-based membranes prepared by using DGD $(\square)$ and DMP $(\Delta)$, respectively, as the plasticizer. 
brane, with a slope of $50 \mathrm{mV}$ per decade for $10^{-5}$ to $10^{-2} \mathrm{M} \mathrm{NH}_{4}{ }^{+}$, were inferior to those of PVCbased membranes.

In an effort to improve the performance of the polyimide matrix membrane, several membranes were prepared by further incorporating lipophilic additives or other plasticizers in addition to nonactin and polyimide (this time, PI-2590-D encapsulant was used). Increasing the amount of lipophilic additive (KTpClPB) in the membrane phase increased the response slope of the resulting PI-2590$\mathrm{D}$ matrix membranes (see Fig. 2). The response was further improved when the membrane was prepared by using an additional plasticizer, dipentyl phthalate (DPP), previously used for the preparation of $\mathrm{NH}_{4}{ }^{+}$-selective polymer membranes [15]. Although incorporating DPP into the PI-2590-D matrix led to a less transparent membrane, the resulting membrane retained its high mechanical strength and homogeneity. As seen in Fig. 2, the response of the membrane was greatly enhanced by using the mixed plasticizer (DGD/ DPP; $50 \mu \mathrm{l} / 50 \mu \mathrm{l})$ and KTpClPB $(0.67 \mathrm{mg})$, along with nonactin $(2 \mathrm{mg}$ ) and PI-2590-D (600 mg).

The potentiometric response of the optimized $\mathrm{NH}_{4}{ }^{+}$-selective PI-2590-D matrix membrane was compared with those of PVC-based membranes. Figure 3 shows that PVC-based membranes prepared using DGD as the plasticizer had significantly degraded response toward $\mathrm{NH}_{4}{ }^{+}$(slope of $47.25 \mathrm{mV}$ per decade over the range $10^{-5}$ to $10^{-1} \mathrm{M} \mathrm{NH}_{4}^{+}$. However, the optimized PI-2590$D$ membranes exhibited nearly the same response slope ( $56.5 \mathrm{mV}$ per decade) as those found for the conventional PVC-based membrane $(56.9 \mathrm{mV}$ per decade). In addition, the selectivity coefficients $\left(\log k \mathrm{NH}_{4} \mathrm{Oot}_{4}, j\right.$ ) obtained for the PI-2590-D matrix

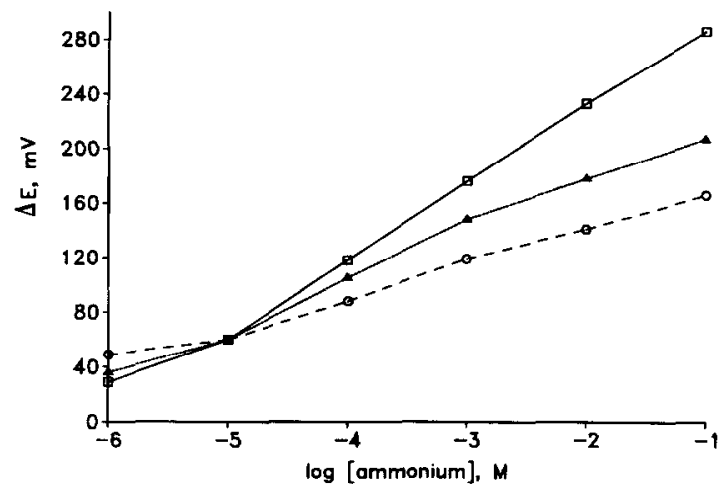

Fig. 2. Potentiometric ammonium response of PI-2590-D matrix membranes having three different compositions: $(O) \mathrm{KT}$ pClPB $(0.67 \mathrm{mg})$, DGD $(100 \mu l) ;(\Delta)$ KTpCIPB $(1.34 \mathrm{mg})$, DGD (100 $\mu \mathrm{l}) ;(\square)$ KTPCIPB (0.67 mg), DGD/DPP ( $50 \mu \mathrm{l} /$ $50 \mu$ l). Each membrane was prepared using $2 \mathrm{mg}$ of nonactin and $600 \mathrm{mg}$ of PI-2590-D polyimide encapsulant.

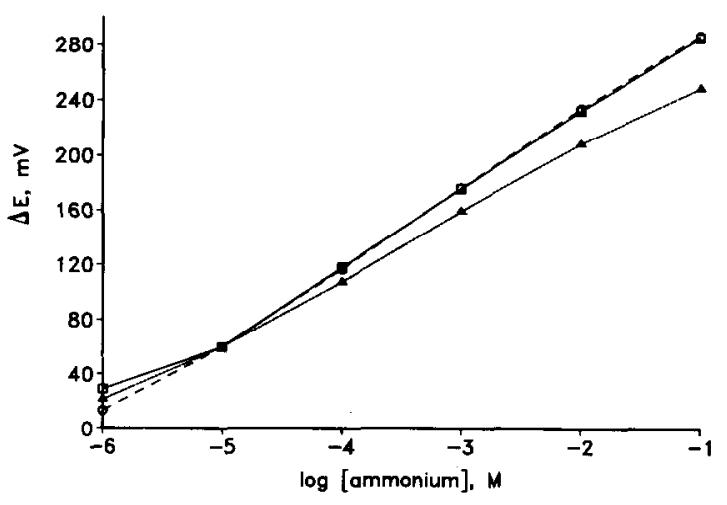

Fig. 3. Potentiometric ammonium response of PVC and PI2590-D matrix membranes doped with nonactin: $(\triangle)$ PVC/ DGD; (O) PVC/DBS; ( $\square$ ) PI-2590-D/DGD/DPP/KTpCIPB.

membrane $\left(-0.9\right.$ for $\mathrm{K}^{+} ;-2.7$ for $\left.\mathrm{Na}^{+}\right)$were similar to those for PVC-based membranes $(-0.9$ for $\mathrm{K}^{+} ;-2.9$ for $\mathrm{Na}^{+}$).

The polyimide matrix was further evaluated by preparing $\mathrm{Ca}^{2+}$-selective membranes. For this, ETH 129 ( $2 \mathrm{mg}$ ) was incorporated with KTpCIPB $(1.2 \mathrm{mg})$, DGD $(66 \mu \mathrm{l})$, and polyimide (PI; $66 \mathrm{mg}$ ). Figure 4 compares the response of the PI matrix membrane to those of conventional PVC and commercial Orion liquid-type $\mathrm{Ca}^{2+}$-selective electrodes. The performance of these electrodes is seen to be comparable except for a slightly higher detection limit for the polyimide matrix membrane.

As expected, the PI membranes exhibited much better adhesion to the silicon nitride surface than did PVC membranes (see Fig. 5). Detachment of the ten PVC membranes occurred over a range of 25 to $60 \mathrm{~min}$, with the median time being $31 \mathrm{~min}$. Five PI membranes detached almost in unison between $9 \mathrm{~h} 37 \mathrm{~min}$ and $9 \mathrm{~h} 40 \mathrm{~min}$, and the remaining five remained intact when the test was

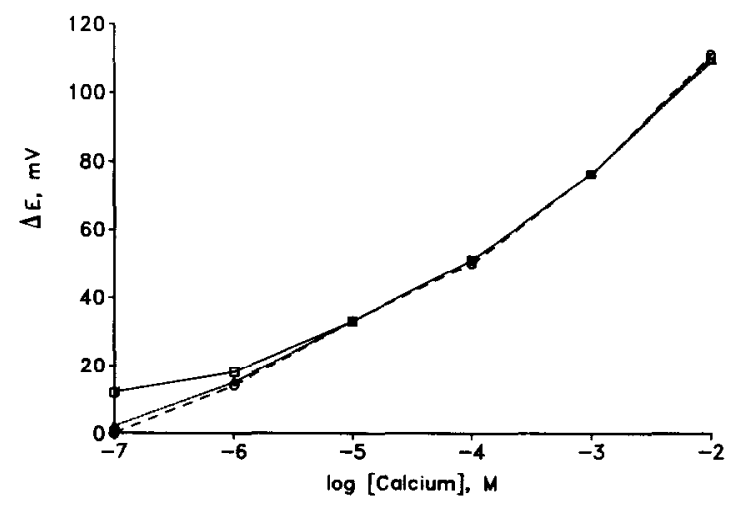

Fig. 4. Potentiometric calcium response of PI and PVC matrix membranes doped with ETH129, and an Orion calcium-selective electrode: ( $\square)$ PI; $(\Delta)$ Orion, (O) PVC. 


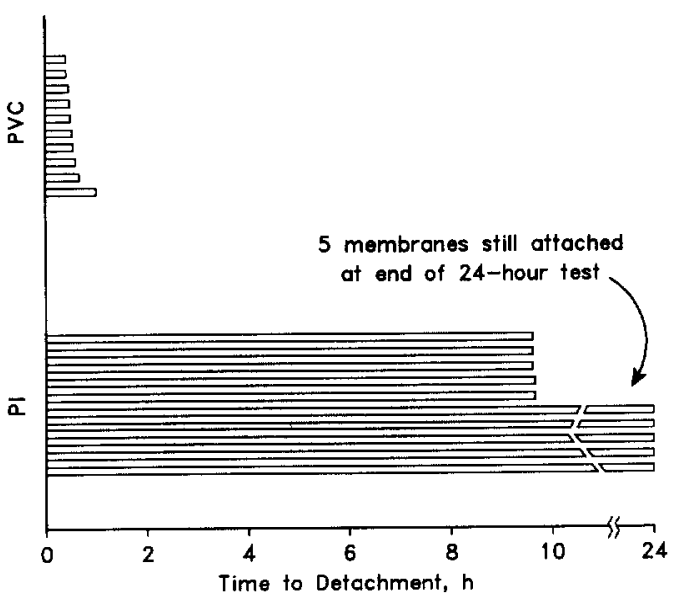

Fig. 5. Accelerated adhesion test of conventional PVC matrix and polyimide matrix membranes on $\mathrm{Si}_{3} \mathrm{~N}_{4}$, showing time to detachment for ten membranes of each type.

terminated after $24 \mathrm{~h}$. The median PI membrane lifetime in this test was, therefore, more than 25 times longer than that of PVC membranes.

In evaluating a variety of membrane materials in the ultrasonic bath accelerated lifetime test, we have observed a correlation between adhesion time and compliance of the membrane. The ultrasonic vibrations are more effective at detaching rigid membranes which are able to absorb the ultrasonic energy through elastic deformation. Comparisons of dissimilar membrane materials with this method, therefore, will favor the softer material. Since PI membranes are harder than PVC membranes, the comparison given can be considered as worst-case for PI membranes. Future studies will evaluate true membrane lifetimes, including degradation of electrochemical properties through such effects as ionophore loss.

\section{Conclusions}

This work has demonstrated the feasibility of using polyimide as the polymer matrix of ionselective membranes. Two plasticizers have been identified which are compatible with polyimide. Membrane compositions for ammonium- and calcium-selective membranes are reported, for which potentiometric slope and selectivity are comparable to those of PVC-based membranes at ion concentrations above $1 \mathrm{ppm}$. The potentiometric response of PI matrix membranes has been tested only in electrode bodies. Our ongoing work involves development of a wafer-level deposition process for such PI membranes, and evaluation of these membranes on solid-state sensor chips.
We have also confirmed the expected improvement in adhesion of plasticized PI membranes compared to PVC membranes; it is hoped that this characteristic will help extend the lifetimes of chemical microsensors. Further studies are needed for complete evaluation of these membranes. Based on this preliminary work, though, polyimide should be considered as one of the material alternatives for making permselective membranes on solid-state chemical sensors.

\section{Acknowledgements}

This work is supported by Fleck Controls, Inc., Brookfield, WI 53008-1006. We express also appreciation to Professor Mark E. Meyerhoff for kindly allowing us use of his laboratory facilities.

\section{References}

1 R. B. Brown, An integrated multiple-sensor chemical transducer, Ph.D. Dissertation, University of Utah, June 1985.

2 T. Satchwill and D. J. Harrison, Synthesis and characterization of new polyvinylchloride chloride membranes for enhanced adhesion on electrode surfaces, $J$. Electroanal. Chem., 202 (1986) 75-81.

3 G. J. Moody, J. D. R. Thomas and J. M. Slater, Modified poly(vinyl chloride) matrix membranes for ion-fieid effect transistor sensors, Analyst, 113 (1988) 1703-1707.

$4 \mathrm{G}$. Blackburn and J. Janata, The suspended mesh ion selective field effect transistor, J. Electrochem. Soc., 129 (1982) 2580-2584.

5 A. K. Covington and P. D. Whalley, Recent advances in microelectronic ion-sensitive devices, J. Chem. Soc., Faraday Trans. I, 82 (1986) 1209-1215.

6 O. J. Prohaska, F. Kohl, P. Goiser, F. Olcaytug, G. Urban, A. Jachimowicz, K. Pirker, W. Chu, M. Patil, J. LaManna and R. Vollmer, Multiple chamber-type probe for biomedical applications, Proc. 4th Int. Cont. SolidState sensors and Actuators (Transducers '87), Tokyo, Japan, June 2-5, 1987, pp. 812-815.

7 H. Blennemann, L. Bouse, L. Bowman and J. D. Meindl, Silicon chemical sensors with microencapsulation of ionselective membranes, Proc. 4th Int. Conf. Solid-State Sensors and Actuators (Transducers '87), Tokyo, Japan, June $2-5,1987$, pp. $723-725$.

8 M. Fujihara, M. Fukui and T. Osa, Chemically modified parylene gate field effect transistors, J. Electroanal. Chem., 106 (1980) 413-418.

9 G. J. Moody, J. M. Slater and J. D. R. Thomas, Membrane design and photocuring encapsulation of flatpack based ion-sensitive field effect transistors, Analyst, 113 (1988) 103-108.

10 C. C. Wen, I. Lauks and J. N. Zemel, Valinomycin-doped photoresist layers for potassium ion sensing, Thin Solid Films, 70 (1980) 333-340.

11 D. Ammann, W. E. Morf, P. Anker, P. C. Meiser, E. Pretsch and W. Simon, Neutral carrier based ion-selective electrodes, Ion-Selective Electrode Rev., 5 (1983) 3-92.

12 A. Craggs, G. J. Moody and J. D. R. Thomas, PVC 
matrix membrane ion-selective electrodes, J. Chem. Educ., 51 (1974) 541-544.

13 U. Schefer, D. Ammann, E. Pretsch, U. Oesch and W. Simon, Neutral carrier based $\mathrm{Ca}^{2+}$-selective electrode with detection limit in the sub-nanomolar range, Anal. Chem., 186 (1986) 2282-2285.

14 G. G. Guilbault, R. A. Durst, M. S. Frant, H. Freiser, E.
H. Hansen, T. S. Light, E. Punger, G. Rechnitz, N. M. Rice, T. J. Rohm, W. Simon and J. D. R. Thomas, Recommendations for nomenclature of ion-selective electrodes, Pure Appl. Chem., 48 (1976) 127-131.

15 G. S. Cha and M. E. Meyerhoff, Potentiometric ion- and bio-selective electrodes based on asymmetric cellulose acetate membranes, Talanta, 36 (1989) 271-278. 